\title{
Chronic angiotensin receptor activation promotes hepatic triacylglycerol accumulation during an acute glucose challenge in obese-insulin-resistant OLETF rats
}

\author{
Jose A. Godoy-Lugo $\mathbb{1}^{1} \cdot$ Max A. Thorwald ${ }^{1,5} \cdot$ David Y. Hui ${ }^{2} \cdot$ Akira Nishiyama $^{3} \cdot$ Daisuke Nakano $^{3}$. \\ Jose G. Soñanez-Organis ${ }^{4} \cdot$ Rudy M. Ortiz ${ }^{1}$
}

Received: 6 April 2021 / Accepted: 18 July 2021 / Published online: 29 July 2021

(c) The Author(s) 2021

\begin{abstract}
Purpose Angiotensin receptor blockers (ARBs) can ameliorate metabolic syndrome (MetS)-associated dyslipidemia, hepatic steatosis, and glucose intolerance, suggesting that angiotensin receptor (AT1) over-activation contributes to impaired lipid and glucose metabolism, which is characteristic of MetS. The aim of this study was to evaluate changes in the lipid profile and proteins of fatty acid uptake, triacylglycerol (TAG) synthesis, and $\beta$-oxidation to better understand the links between AT1 overactivation and non-alcoholic fatty liver disease (NAFLD) during MetS.

Methods Four groups of 25-week-old-rats were used: (1) untreated LETO, (2) untreated OLETF, (3) OLETF + angiotensin receptor blocker (ARB; $10 \mathrm{mg}$ olmesartan $/ \mathrm{kg} / \mathrm{d} \times 8$ weeks) and (4) OLETF \pm ARB (MINUS; $10 \mathrm{mg}$ olmesartan $/ \mathrm{kg} / \mathrm{d} \times$ 4 weeks, then removed until dissection). To investigate the dynamic shifts in metabolism, animals were dissected after an oral glucose challenge (fasting, 3 and $6 \mathrm{~h}$ post-glucose).

Results Compared to OLETF, plasma total cholesterol and TAG remained unchanged in ARB. However, liver TAG was 55\% lesser in ARB than OLETF, and remained lower throughout the challenge. Basal CD36 and ApoB were 28\% and 29\% lesser, respectively, in ARB than OLETF. PRDX6 abundance in ARB was 45\% lesser than OLETF, and it negatively correlated with liver TAG in ARB.

Conclusions Chronic blockade of AT1 protects the liver from TAG accumulation during glucose overload. This may be achieved by modulating NEFA uptake and increasing TAG export via ApoB. Our study highlights the contributions of AT1 signaling to impaired hepatic substrate metabolism and the detriments of a high-glucose load and its potential contribution to steatosis during MetS.
\end{abstract}

Keywords Angiotensin receptor blocker $\cdot$ ARB, Insulin resistance $\cdot$ Liver steatosis $\cdot$ Metabolic syndrome $\cdot$ NAFLD

Jose A. Godoy-Lugo

jgodoy4@ucmerced.edu

1 School of Natural Sciences, University of California, Merced, CA, USA

2 Pathology and Laboratory Medicine, University of Cincinnati, Cincinnati, OH, USA

3 Department of Pharmacology, Kagawa University Medical School, Kagawa, Japan

4 Universidad de Sonora, Departamento de Ciencias Químico Biológicas y Agropecuarias, Navojoa, Sonora, Mexico

5 Present address: Leonard Davis School of Gerontology, University of Southern California, Los Angeles, CA, USA

\section{Introduction}

Non-alcoholic fatty liver disease (NAFLD) afflicts $25 \%$ of the world's population and is projected to increase due to the increasing prevalence of diabetes and obesity $[1,2]$. The metabolic syndrome (MetS) comprises several disorders that raise the risk of NAFLD [3]. MetS is identified when at least three of the following criteria are present simultaneously: (a) abdominal obesity, (b) elevated circulating triacylglycerol (TAG), (c) reduced HDL cholesterol levels, (d) hypertension, and (e) increased fasting glucose \pm insulin resistance [4]. Obesity and insulin resistance are factors that contribute to the development of MetS [3].

Inappropriately elevated angiotensin II (Ang II) and over-activation of its target receptor, AT1, contribute to 
impaired hepatic lipid metabolism [5], development of fatty liver [6], and the development of insulin resistance [7-9]. Thus, elevated Ang II is regarded as a risk factor for NAFLD [10]. Chronic infusion of Ang II increased circulating insulin, TAG, NEFA, and liver TAG synthesis in rats [11, 12]. Ang II-mediated activation of AT1 may overload the liver by increasing plasma NEFA, decreasing fatty acid oxidation, and promoting de novo lipogenesis [5]. Angiotensin receptor blockers (ARB) displace Ang II from its receptor [13], inhibiting Ang II signaling. While ARBs are widely used to ameliorate MetS-related hypertension [14], they also improve markers of inflammation $[15,16]$, components of the lipid profile, and hepatic lipid accumulation in animal studies and clinical trials [17-21]. However, the mechanisms contributing to these improvements are not fully understood, especially in relationship to AT1 signaling.

The sequestration of hepatic NEFA is mainly dependent on membrane fatty acid transporters, which include the fatty acid transport protein (FATP) family [22], cluster of differentiation 36 (CD36), and caveolins $[23,24]$. In the liver, FATP5 and FATP2 are the most abundantly expressed of the 6 members of the FATP family. Knockdown [25] and knockout (KO) [26] of FATPs ameliorated hepatic steatosis. These studies also demonstrated a positive correlation between FATP levels and NEFA uptake in the liver [25, 26]. Hepatic expression of CD36 is typically low but increases with fatty liver [27, 28]. A positive correlation between plasma insulin levels and hepatic CD36 expression was observed in insulin-resistant patients with steatosis [29].

Recent studies suggest that peroxiredoxin 6 (PRDX6) may provide an anti-steatotic effect during fatty liver disease, mainly through elimination of oxidants [30]. PRDX6 is a special member of its family because it is bifunctional, having both peroxidase and phospholipase A2 activity [31]. PRDX6 protected the liver against damage and mitochondrial dysfunction induced by oxidative stress during ischemia-reperfusion [32]. NAFLD is associated with excessive oxidant production, which is linked with impaired mitochondrial function [33]. Yet, studies addressing hepatic PRDX6 changes in a MetS model do not exist.

Hepatic lipid accumulation is the hallmark of NAFLD [3, 34], which may result from an imbalance between lipid acquisition and their disposal or oxidation [27]. Nonetheless, the mechanisms that promote the development of steatosis, and the potential AT1 signaling involved, are not completely understood $[27,35]$. In the present study we investigated liver lipid substrates and proteins for NEFA uptake, TAG and VLDL cholesterol synthesis, and fatty acid oxidation in the liver of rats treated with ARB and their changes in response to an acute glucose challenge, as well as the potential effects of the removal of ARB treatment.
Table 1 Mean $( \pm \mathrm{SD})$ end of study body mass and plasma angiotensin II

\begin{tabular}{|c|c|c|c|c|}
\hline & LETO & OLETF & ARB & MINUS \\
\hline $\begin{array}{l}\text { Body } \\
\text { Mass (g) }\end{array}$ & $465 \pm 29$ & $610 \pm 31^{\mathrm{a}}$ & $598 \pm 36^{\mathrm{a}}$ & $602 \pm 28^{\mathrm{a}}$ \\
\hline $\begin{array}{l}\text { Plasma AngII } \\
\text { (fmol/ml) }\end{array}$ & $58.9 \pm 3.1$ & $68.6 \pm 11.6$ & $211.3 \pm 27.9^{\mathrm{a}, \mathrm{b}}$ & $65.2 \pm 10.2^{c}$ \\
\hline
\end{tabular}

${ }^{\text {a }}$ Significant difference from LETO $(P<0.05)$

${ }^{\mathrm{b}}$ Significant difference from OLETF $(P<0.05)$

${ }^{\mathrm{c}}$ Significant difference from ARB $(P<0.05)$

\section{Methods}

All experimental procedures were reviewed and approved by the institutional animal care and use committees of the Kagawa Medical University (Japan) and the University of California, Merced (USA). Phenotypical data (i.e., body mass, oral glucose tolerance test curves, insulin levels, and blood pressure) has been previously published [36] with that study focused on redox signaling in the heart in response to a glucose challenge. The current study is unique and complements the previous one by extending the examination of the dynamic changes in hepatic lipid metabolism in response to a glucose challenge, which represents a nutrient overload that may appear in Western diets [37, 38]. We have included previously published [36] body mass and plasma angiotensin II levels (Table 1) to better illustrate our model and the efficacy of the ARB treatment. Additionally, we examine the potential detriments of non-compliance with ARB treatment after the initial treatment has stopped (legacy effect) [39]. Low compliance has proven to be a challenge when treating patients with hypertension, dyslipidemia, and diabetes $[40,41]$ and assessing ARB-legacy effects may provide a better understanding of the detriments to removal of treatment $[42,43]$.

In this study, we approach the obtained results from two perspectives: (1) the static outcomes following the chronic treatment with ARB and its removal (potential legacy effect) and (2) the dynamic response to a glucose challenge following chronic treatment with ARB and its removal (non-compliance). In all cases, comparisons are made to a lean, strain-control.

\section{Animals}

OLETF rats are reported to develop insulin resistance and hyperglycemia by 17 weeks of age [44, 45]. For that reason, male, age matched, 17-week-old, lean strain-control Long Evans Tokushima Otsuka (LETO; $428 \pm 8 \mathrm{~g}$ ) and obese Otsuka Long Evans Tokushima Fatty (OLETF; $536 \pm 6 \mathrm{~g}$ ) rats (Japan SLC Inc., Hamamatsu, Japan) were used. Rats 
were assigned to the following groups ( $n=5-7$ animals/ group/time point): (1) untreated LETO, (2) untreated OLETF, (3) OLETF + angiotensin receptor blocker (ARB; $10 \mathrm{mg}$ olmesartan $/ \mathrm{kg} / \mathrm{d} \times 8$ weeks) [46], and (4) OLETF \pm ARB (MINUS; $10 \mathrm{mg}$ olmesartan $/ \mathrm{kg} / \mathrm{d} \times 4$ weeks, then removed the last 4 wks prior to dissection). ARB (DaiichiSankyo, Tokyo, Japan) was administered by oral gavage suspended in carboxymethyl cellulose (CMC) to conscious rats and untreated rats were gavaged with CMC only. Animals were maintained in groups of two to three animals per cage, given access to water and standard laboratory chow (MF; Oriental Yeast Corp., Tokyo, Japan), and maintained under controlled temperatures $\left(23-24^{\circ} \mathrm{C}\right)$ and humidity $(\sim 55 \%)$ with a light-dark cycle of $12-12 \mathrm{~h}$.

For dissections, the 25-week-old rats were fasted for $12 \mathrm{~h} \pm 15 \mathrm{~min}$. To investigate the dynamic response to a glucose challenge, animals were dissected at baseline (T0, fasting) and after 3 (T3) and $6 \mathrm{~h}$ (T6) after a glucose load by gavage ( $2 \mathrm{~g}$ glucose $/ \mathrm{kg}$ mass). Comparisons of the T0 (fasting baseline) data from each group characterized the static changes in response to chronic ARB treatment and its removal. The glucose challenge was performed to evaluate the acute, dynamic changes in metabolism and cellular responses. Initiation of the overnight fasts and the glucose gavages were staggered to meet the exact dissections timepoints. Animals were decapitated and trunk blood collected in vials containing EDTA (Sigma-Aldrich, EDS) and proteinase inhibitor cocktail (Sigma-Aldrich, P2714). Livers were snap frozen in liquid nitrogen and kept at $-80^{\circ} \mathrm{C}$ until analyzed.

\section{Biochemical analyses \& markers of hepatic NAFLD}

Insulin, glucose, plasma total cholesterol (TC), TAG, and NEFA concentrations as well as collagen type IV (COL-4) were measured using the commercially available reagents: Insulin Rat ELISA Kit (Thermo Fisher Scientific, ERINS), Autokit glucose (Fujifilm Wako Diagnostics, 997-03001), Total Cholesterol E (Fujifilm Wako Diagnostics, 99902601), L-Type Triglyceride M (Fujifilm Wako Diagnostics, 994-02891 and 990-02991), HR Series NEFA-HR (2) (Fujifilm Wako Diagnostics, 999-34691, 995-34791, 991-34891, and 993-35191), and CIV ELISA (MyBioSource, MBS732756), respectively, following manufacturer's instructions. All samples were analyzed in duplicate and only accepted values that fell within a percent coefficient of variability of less than $10 \%$ for all measurements.

\section{Western blot analyses}

A $25 \mathrm{mg}$ piece of frozen liver was homogenized in phosphate buffer for a two-step extraction of cytoplasm and plasma membrane proteins. Briefly, phosphate buffer (50 $\mathrm{mM}$ potassium phosphate) (Fisher Scientific, P290 and P285) was used to homogenize the liver, then centrifuged at $15,000 \times g$ to recover the supernatant containing the cytoplasmic fraction. Then, $50 \mathrm{mM}$ potassium phosphate buffer $+1 \%$ Triton X-100 (Millipore-Sigma, T8787) was used to homogenize the pellet, which contained the plasma membrane fraction. The pellet homogenate was centrifuged at $15,000 \times g$, and the plasma membrane contents recovered from the supernatant. The buffers contained 3\% protease inhibitor cocktail (Sigma-Aldrich, P2714) to help prevent protein degradation. The protein content of the fractions was quantified using the Bradford assay (Bio-Rad Laboratories, 5000203). Total protein $(5-10 \mu \mathrm{g})$ was resolved in $10 \%$ Tris-HCL SDS gels. Proteins were electroblotted onto $0.45-\mu \mathrm{m}$ polyvinyl difluoride (PVDF) (Millipore-Sigma, IPVH00010) membranes by semi-wet transfer using the Mini Gel Tank and Blot Module Set (Invitrogen, NW2000). Intercept blocking buffer (Li-Cor, 927-60001, 927-70001) was used to block the membranes then incubated $16 \mathrm{~h}$ with the corresponding primary antibody (diluted 1:1,0001:2,000) against glycerol-3-phosphate acyltransferase 1 (GPAM) (abcam, ab69990), FATP5 (Invitrogen, PA542028), diacylglycerol O-acyltransferase 1 (DGAT1) (Thermo Fisher, PA5-79150), ApoB (Apolipoprotein B) (Thermo Fisher, PA5-86950), CD36 (Thermo Fisher, PA116813), FATP2 (Thermo Fisher, PA5-30420), carnitine palmitoyl transferase I (CPT1A) (Proteintech, 15184-1-AP), Peroxisomal acyl-coenzyme A oxidase 1 (Acox1) (Thermo Fisher, PA5-76341), PRDX6 (Proteintech, 13585-1-AP), and $\beta$ actin (Cell Signaling Technology, 3700S) (diluted 1:5,000). Membranes were washed, incubated with IRDye 800CW anti-rabbit (Li-Cor, 926-32213) and/or 680RD donkey anti-mouse IgG secondary antibodies (Li-Cor, 92668072) (diluted 1:20,000), and rewashed. Blots were visualized using the Odyssey system (Li-Cor) and quantified using the Image Studio Lite ver. 5.2 (Li-Cor) using $\beta$ actin as a loading control. Plasma membrane and cytosolic fractions were tested for purity against $\mathrm{Na}^{+} / \mathrm{K}^{+}$ATPase antibody (Abcam, ab76020) (diluted 1:40,000) and alpha tubulin (Abcam, ab52866) (diluted 1:40,000), respectively.

\section{Statistics}

Data was tested for normality using the Shapiro-Wilk test [47]. Means \pm standard deviation (SD) were compared by two-way ANOVAs when analyzing datasets with all timepoints (T0, T3, T6) and groups, and one-way-ANOVA when analyzing datasets with only basal levels (T0 only). Means were considered significantly different at $p<0.05$ using Tukey's HSD. Correlations were calculated using the Pearson $\mathrm{r}$ coefficient $[48,49]$, using the means and SD obtained from each group and timepoint, computed using 
the displayed individual values in each figure. Area under the curve (AUC) analyses were calculated using the area under the concentration curve in batch designs [50]. Outliers were calculated and removed using the ROUT test [51] and one outlier was replaced with the mean of the corresponding group [52]. All statistical analyses were performed with GraphPad Prism 8.4.3 software (GraphPad Prism, La Joya, CA).

\section{Results}

To better recognize the difference between static (i.e., chronic ARB treatment reflected by differences at T0) and dynamic (i.e., during the acute glucose load) responses, the results were separated into two sections contained within each subheading, designated respectively to the response.

\section{ARB normalized the secretion of insulin in response to glucose}

Previous studies have shown that ARBs decrease plasma glucose [53, 54] and report improvements in insulin resistance [55], yet other studies report no changes [56, 57]. Therefore, to better address the existing discrepancies in the literature, we measured plasma glucose and insulin statically over time and dynamically in response to the glucose load.

\section{Static Changes}

Basal circulating glucose levels were $34 \%, 30 \%$, and $46 \%$ greater in OLETF, ARB, and MINUS, respectively, than LETO (Fig. 1A). While levels in MINUS were 22\% and $29 \%$ greater than OLETF and ARB, respectively (Fig. 1A). Basal plasma insulin was $80 \%$ and $73 \%$ greater in OLETF and MINUS, respectively, compared to LETO (Fig. 1C). The basal glucose-to-insulin ratios were not different among the groups (Fig. 1E).

\section{Dynamic changes}

During the acute glucose challenge, circulating glucose levels were $40 \%$ and $30 \%$ greater in OLETF, $36 \%$ and $37 \%$ greater in ARB, and $31 \%$ and $55 \%$ greater in MINUS, when compared to LETO at T3 and T6, respectively (Fig. 1A). Levels in MINUS at T6 were $18 \%$ and $12 \%$ greater than OLETF and ARB, respectively (Fig. 1A). Mean glucose AUC was $38 \%, 38 \%$, and $48 \%$ greater in OLETF, ARB and MINUS, respectively, when compared to LETO, whereas mean AUC in MINUS was $8 \%$ greater than OLETF and ARB (Fig. 1B).
At T3, plasma insulin levels were $183 \%$ greater in OLETF compared to LETO, and $89 \%$ and $74 \%$ lesser in ARB and MINUS, respectively, compared to OLETF (Fig. 1C). At T6, levels were $75 \%$ and $145 \%$ greater in OLETF and MINUS, respectively, when compared to LETO (Fig. 1C). Plasma insulin levels were $30 \%$ and $50 \%$ lesser in ARB compared to OLETF and MINUS, respectively (Fig. 1C). Mean insulin AUC was $140 \%, 52 \%$, and $99 \%$ greater in OLETF, ARB, and MINUS, respectively, when compared to LETO (Fig. 1D). Mean AUCs were $37 \%$ and $17 \%$ lesser in ARB and MINUS, respectively, compared to OLETF, whereas MINUS AUC was $31 \%$ greater compared to ARB (Fig. 1D).

At T3, the glucose-to-insulin ratio was 50\% lesser in OLETF compared to LETO, while ratios in ARB and MINUS were $45 \%$ and $37 \%$ greater than OLETF (Fig. 1E). At T6, ratios in OLETF and MINUS were $43 \%$ and 53\% lesser than LETO, while the ARB ratio was $39 \%$ greater than OLETF. The ratio on MINUS remained $49 \%$ lesser than ARB (Fig. 1E). Mean glucose-to-insulin ratio AUC was $38 \%, 9 \%$, and $15 \%$ lesser in OLETF, ARB, and LETO, respectively, compared to LETO (Fig. 1F). Mean ratios in ARB and MINUS were $32 \%$ and $15 \%$ greater, respectively, compared to OLETF, while ratio on MINUS remained 25\% lesser compared to ARB (Fig. 1F).

Contrasting with other studies [53, 54], ARB did not change the glucose levels, yet it lowered insulin levels during the glucose challenge and ameliorated the glucoseto-insulin ratio as seen previously $[55,56]$.

\section{ARB increased plasma NEFAs without changing TAG levels}

COL-4 has been shown to accurately detect NAFLD with levels representing the degree of severity [58, 59].

\section{Static Changes}

COL-4 levels were $178 \%$ and $157 \%$ greater in OLETF and MINUS, respectively, compared to LETO (Fig. 2A). Levels were $155 \%$ lesser in ARB compared to OLETF (Fig. 2A). Levels in MINUS were intermediate between ARB and OLETF with levels $135 \%$ greater than ARB (Fig. 2A). These results suggest that liver fibrosis is decreased with $\mathrm{ARB}$, while the removal of the treatment led to an intermediary phenotype associated with loss of most of the ARB benefits.

While it is known that ARB treatment improves circulating NEFA, TAG, and TC [12, 17, 60], these variables were measured here to better interpret the changes as they relate to the changes in the proteins associated with their metabolism and to ascertain the effects of the glucose 


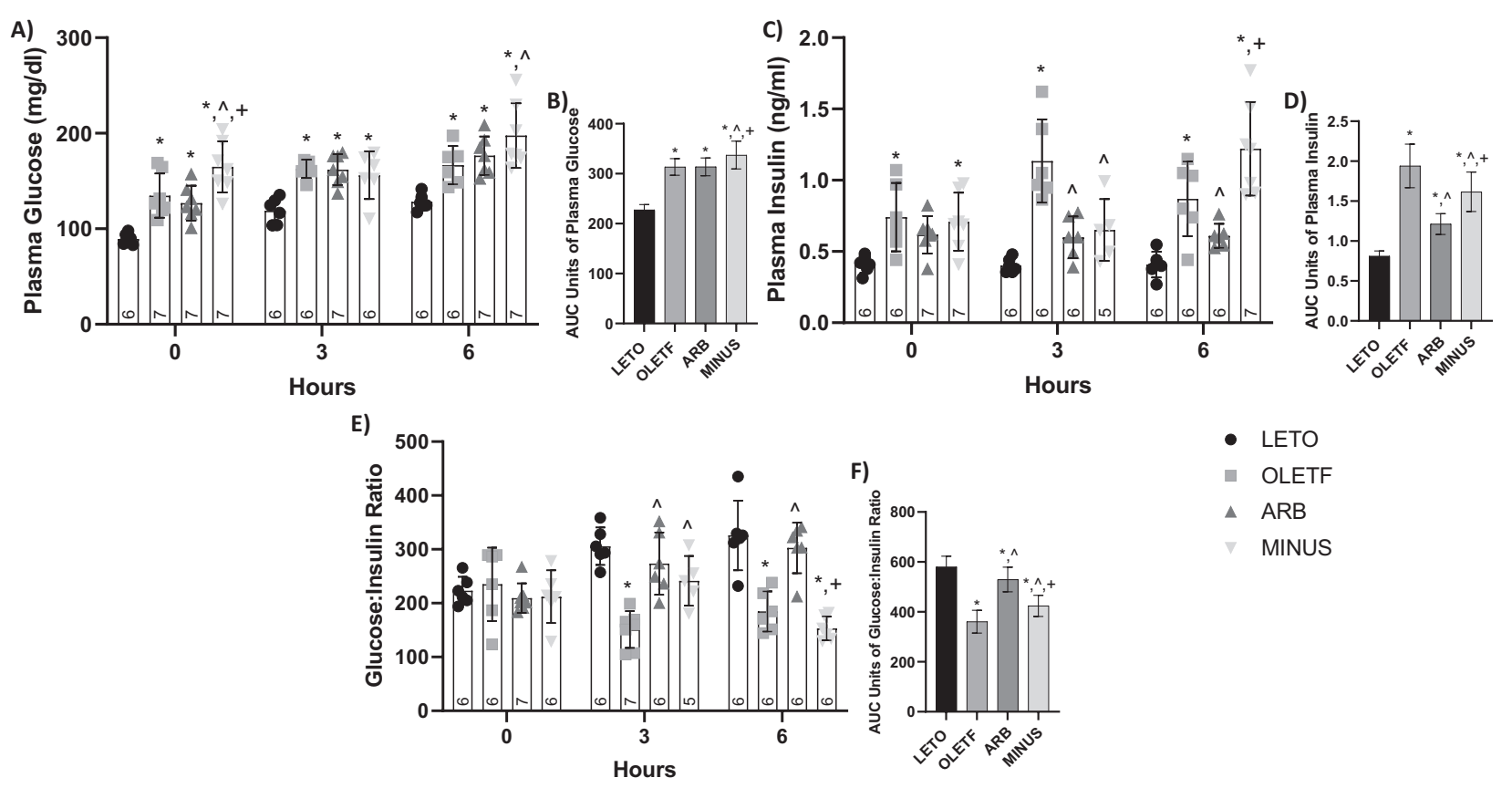

Fig. 1 ARB decreased plasma insulin after a glucose load. Mean \pm SD values of plasma glucose (A), AUC of plasma glucose (B), plasma insulin (C), AUC of plasma insulin (D), glucose:insulin ratio $(\mathbf{E})$, and AUC of glucose:insulin ratio (F), during the glucose challenge in Long Evans Tokushima Otsuka (LETO), Otsuka Long Evans Tokushima
Fatty (OLETF), OLETF + ARB (ARB; ARB x 8 weeks), and OLETF \pm ARB (MINUS, ARB x 4 weeks, then removed $\times 4$ weeks) rats. Number at bottom of the bar indicates $\mathrm{n}$ per group. *Significant difference from LETO $(P<0.05)$. ^ Significant difference from OLETF $(P<0.05) .+$ Significant difference from $A R B(P<0.05)$
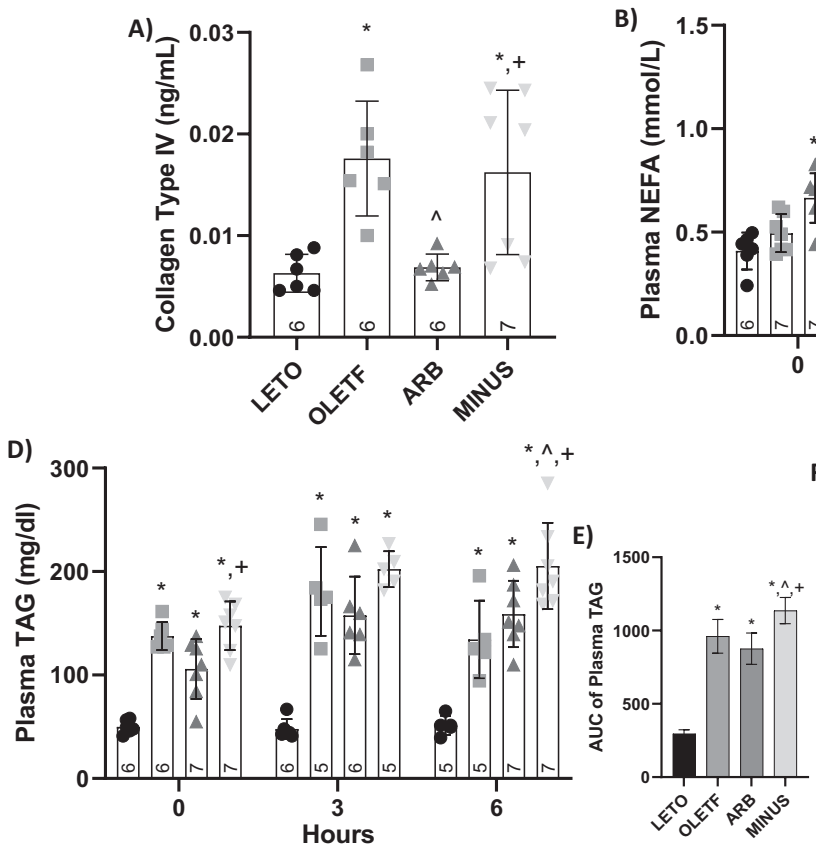

Fig. 2 ARB increased plasma NEFAs without changing TAG levels. Mean \pm SD values of COL-4 (A) plasma NEFA (B), AUC of plasma NEFA (C), plasma triacylglycerol (D), AUC of plasma triacylglycerol $(\mathbf{E})$, plasma total $(\mathbf{F})$ and AUC $(\mathbf{G})$ of total cholesterol, during the glucose challenge in Long Evans Tokushima Otsuka (LETO), Otsuka Long Evans Tokushima Fatty (OLETF), OLETF + ARB (ARB; ARB
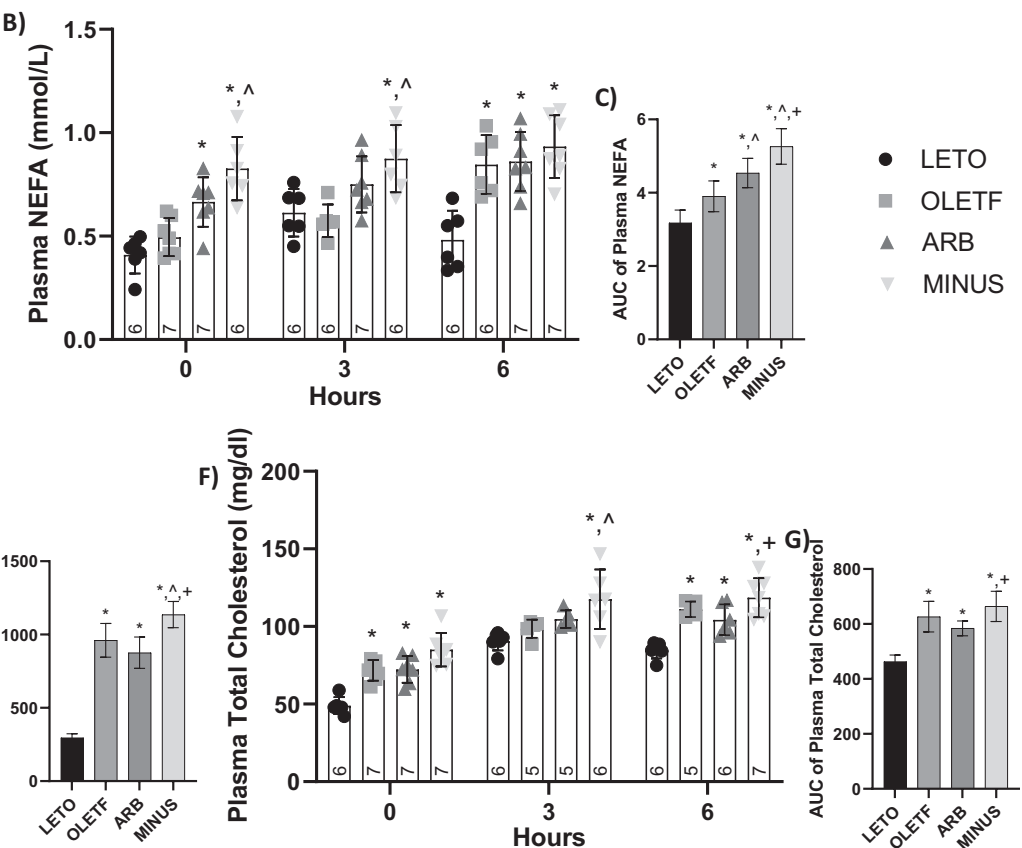

$\mathrm{x} 8$ weeks), and $\mathrm{OLETF} \pm \mathrm{ARB}$ (MINUS, ARB $\mathrm{x} 4$ weeks, then removed $\mathrm{x} 4$ weeks) rats. Number at bottom of the bar number indicates $n$ per group. *Significant difference from LETO $(P<0.05)$. $\wedge$ Significant difference from OLETF $(P<0.05)$. + Significant difference from $A R B(P<0.05)$ 
challenge on lipid metabolism. Static Changes: Basal plasma NEFA was $38 \%$ and $50 \%$ greater in ARB and MINUS, respectively, compared to LETO (Fig. 2B). Plasma TAG was $174 \%, 110 \%$, and $194 \%$ greater in OLETF, ARB and MINUS, respectively, compared to LETO, while MINUS remained $40 \%$ greater than ARB (Fig. 2D). Plasma TC was $47 \%, 47 \%$, and $73 \%$ greater in OLETF, ARB, and MINUS, respectively, compared to LETO (Fig. 2F).

\section{Dynamic changes}

During the glucose challenge, plasma NEFA in MINUS was $43 \%$ and $30 \%$ greater than LETO and OLETF at T3, respectively (Fig. 2B). At T6, levels in OLETF, ARB and MINUS were $76 \%, 79 \%$, and $93 \%$ greater, respectively, compared to LETO (Fig. 2B). Mean plasma NEFA AUC was $17 \%, 42 \%$, and $64 \%$ greater in OLETF, ARB and MINUS, respectively, compared to LETO (Fig. 2C). Mean AUC in ARB and MINUS was 17\% and 29\%, respectively, greater than OLETF, while MINUS remained $14 \%$ greater than ARB (Fig. 2C).

At T3, plasma TAG levels in OLETF, ARB, and MINUS remained 275\%, 227\%, and 320\% greater, respectively, than LETO (Fig. 2D). At T6, levels in OLETF, ARB, and MINUS were $162 \%, 211 \%$, and $302 \%$ greater, respectively, than LETO. While MINUS remained $53 \%$ and $29 \%$ greater compared to the OLETF and ARB groups, respectively (Fig. 2D). Mean plasma TAG AUC was $222 \%, 195 \%$, and $285 \%$ greater in OLETF, ARB, and MINUS, respectively, compared to LETO, while MINUS remained $20 \%$ and $30 \%$ greater than ARB and OLETF, respectively (Fig. 2E).

At T3, plasma TC levels were $23 \%$ and $98 \%$ greater in MINUS than LETO and OLETF, respectively (Fig. $2 \mathrm{~F})$. At T6, levels were $30 \%, 22 \%$, and $38 \%$ greater in OLETF, ARB, and MINUS, respectively, compared to LETO, while MINUS remained $13 \%$ greater than ARB (Fig. 2F). Mean plasma TC AUC was $21 \%, 22 \%$, and $39 \%$ greater in OLETF, ARB, and MINUS, respectively, compared to LETO, and MINUS remained $15 \%$ and $14 \%$ greater than OLETF and ARB, respectively (Fig. 2G).

ARB plasma NEFA AUC was greater than OLETF, while plasma TAG AUC remained unchanged. On the other hand, MINUS AUCs were all greater than ARB, which demonstrates the detrimental effect of treatment removal. AT1 activation triggers mechanisms that induce insulin resistance and hypertension [42], the progression insulin resistance in these animals is exponential during the its youth [44]. In our study, we treated our rats during its early stages of MetS (17 weeks of age) and the more developed stage (21 weeks of age), when the rat presents pronounced hepatic steatosis $[44,61]$. During the initial phase of AT1 blockade ( 4 weeks of treatment), the measured parameters may be ameliorated. Though when the treatment is removed, the impact of Ang II signaling may worsen, acting stronger, which may be explained as a drug-derived rebound effect [62].

\section{ARB protected against the glucose-induced accumulation of hepatic TAG}

We measured part of the hepatic lipid profile to confirm this effect and further investigate it during a glucose challenge.

\section{Static Changes}

Basal liver NEFA was $47 \%$ greater in OLETF than LETO, while ARB was $39 \%$ lesser than OLETF (Fig. 3A). Liver TAG was $1880 \%, 787 \%$, and $670 \%$ greater in OLETF, ARB, and MINUS, respectively, compared to LETO, while levels in ARB and MINUS remained 55\% and 61\% lesser than OLETF (Fig. 3C). Liver TC was 58\% and $64 \%$ greater in OLETF and ARB, respectively, compared to LETO (Fig. 3E).

\section{Dynamic Changes}

During the glucose challenge, liver NEFA levels in ARB and MINUS were $53 \%$ and $81 \%$ greater than LETO at T3, respectively, while MINUS remained $33 \%$ greater than OLETF (Fig. 3A). Mean liver NEFA AUC was 40\%, 38\%, and $62 \%$ greater in OLETF, ARB, and MINUS, respectively, compared to LETO, while MINUS remained $15 \%$ and $17 \%$ greater than OLETF and ARB, respectively (Fig. 3B).

At T3, liver TAG levels were $4790 \%$ and $2760 \%$ greater in OLETF and MINUS, respectively, compared to LETO, and remained $41 \%$ lesser in MINUS compared to OLETF, and $138 \%$ greater than ARB (Fig. 3C). At T6, levels were $2600 \%$ and $1040 \%$ greater in OLETF and MINUS, respectively, compared to LETO, while ARB levels were $72 \%$ lesser than OLETF, and MINUS levels were 70\% greater than ARB (Fig. 3C). Mean liver TAG AUC was $3260 \%, 900 \%$, and $1935 \%$ greater in OLETF, ARB, and MINUS, respectively, compared to LETO. ARB and MINUS mean liver TAG remained $40 \%$ and $70 \%$ lesser, respectively, than OLETF, while MINUS remained 50\% greater than ARB (Fig. 3D).

At T3, liver TC levels were $195 \%, 74 \%$, and $90 \%$ greater in OLETF, ARB and MINUS, respectively, compared to LETO, while ARB and MINUS remained $41 \%$ and $36 \%$ lesser than OLETF, respectively (Fig. 3E). At T6, levels were $159 \%, 76 \%$, and $143 \%$ greater in OLETF, ARB, and MINUS, respectively, compared to LETO. ARB remained $32 \%$ lesser than OLETF and MINUS remained $28 \%$ greater 

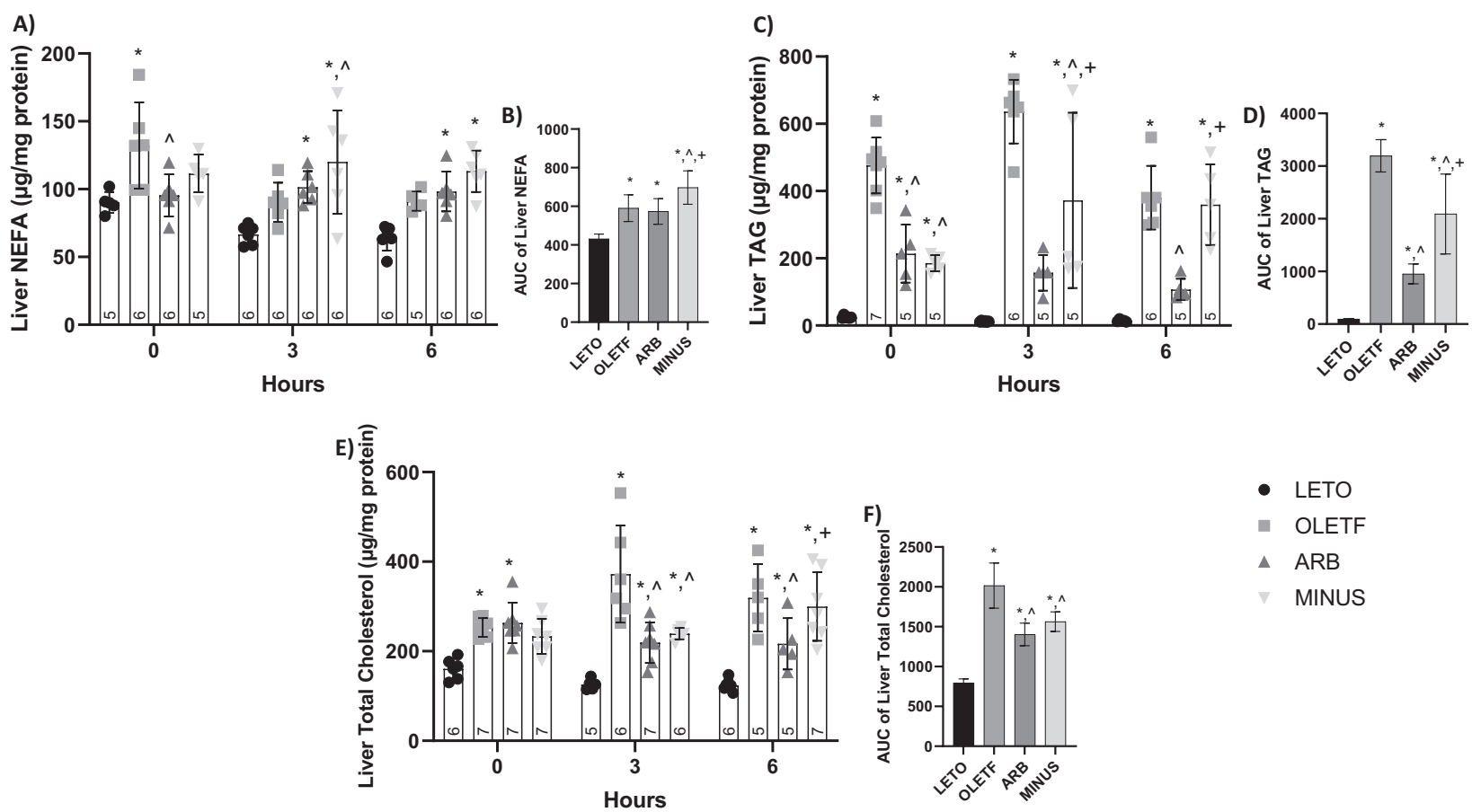

- LETO

- OLETF

$\triangle \mathrm{ARB}$

MINUS

Fig. 3 ARB conferred protection against the glucose-induced increase of hepatic TAG. Mean \pm SD values of liver NEFA (A), AUC of NEFA (B), triacylglycerol (C), AUC of triacylglycerol (D), total cholesterol (E), and AUC of total cholesterol (F), during the glucose challenge in Long Evans Tokushima Otsuka (LETO), Otsuka Long Evans Tokushima Fatty (OLETF), ARB (OLETF + angiotensin receptor

than ARB (Fig. 3E). Mean liver TC AUC was 145\%, 71\%, and $89 \%$ greater in OLETF, ARB, and MINUS, respectively, compared to LETO, while ARB and MINUS remained $30 \%$ and $23 \%$ lesser than OLETF, respectively (Fig. 3F).

ARB improved hepatic NEFA and TAG levels and protected it from TC accumulation during the glucose challenge. Notably, the MINUS group showed ameliorated basal hepatic TAG accumulation; however, during the glucose challenge, TAG and NEFA increased, along with their respective AUC. We attributed this to the re-bound effect [62], as thoroughly discussed above, here MINUS demonstrates more detrimental effects following noncompliance.

\section{ARB decreased basal the abundance of CD36, and $\beta$-oxidation proteins in the liver following the glucose challenge}

Because the liver-specific deletion of AT1 reduces hepatic steatosis [63], we wanted to assess the potential for decreased sequestration of NEFA as a contributing factor. Therefore, we measured the protein abundance of CD36, FATP5, and FATP2, which are the main liver NEFA transporters [22, 64, 65]. blocker $x 8$ weeks), and MINUS (OLETF + angiotensin receptor blocker $\mathrm{x} 4$ weeks, then removed $\mathrm{x} 4$ weeks) rats. Number at bottom of the bar number indicates $n$ per group. *Significant difference from LETO $(P<0.05)$. ^ Significant difference from OLETF $(P<0.05)$. ${ }^{+}$Significant difference from $A R B(P<0.05)$

\section{Static Changes}

Basal CD36 protein abundance was $79 \%, 53 \%$, and $74 \%$ greater in OLETF, ARB and MINUS, respectively, compared to LETO and remained $28 \%$ and $20 \%$ lower in ARB and MINUS, respectively, compared to OLETF (Fig. 4A). CD36 abundance and basal levels of fasting plasma insulin were significantly and positively correlated $(r=0.9836$, $p=0.008$ ) (Fig. 4D). FATP5 protein abundance was 52\%, $62 \%$, and $50 \%$ greater in MINUS than LETO, OLETF, and ARB, respectively (Fig. 4B). FATP2 protein abundance was $46 \%$ and $35 \%$ greater in MINUS compared to OLETF and ARB, respectively (Fig. 4C).

\section{Dynamic changes}

During the glucose challenge, CD36 protein abundance was $70 \%, 75 \%$, and $73 \%$ greater in OLETF, ARB, and MINUS, respectively, compared to LETO at T3 (Fig. 4A). At T6, protein abundance was $80 \%, 75 \%$, and $73 \%$ greater in OLETF, ARB, and MINUS, respectively, compared to LETO (Fig. 4A). While ARB was $25 \%$ greater than OLETF and $28 \%$ lesser than MINUS (Fig. 4A).

At T3, FATP5 protein abundance was $34 \%$ and $44 \%$ greater in MINUS than LETO and ARB, respectively 

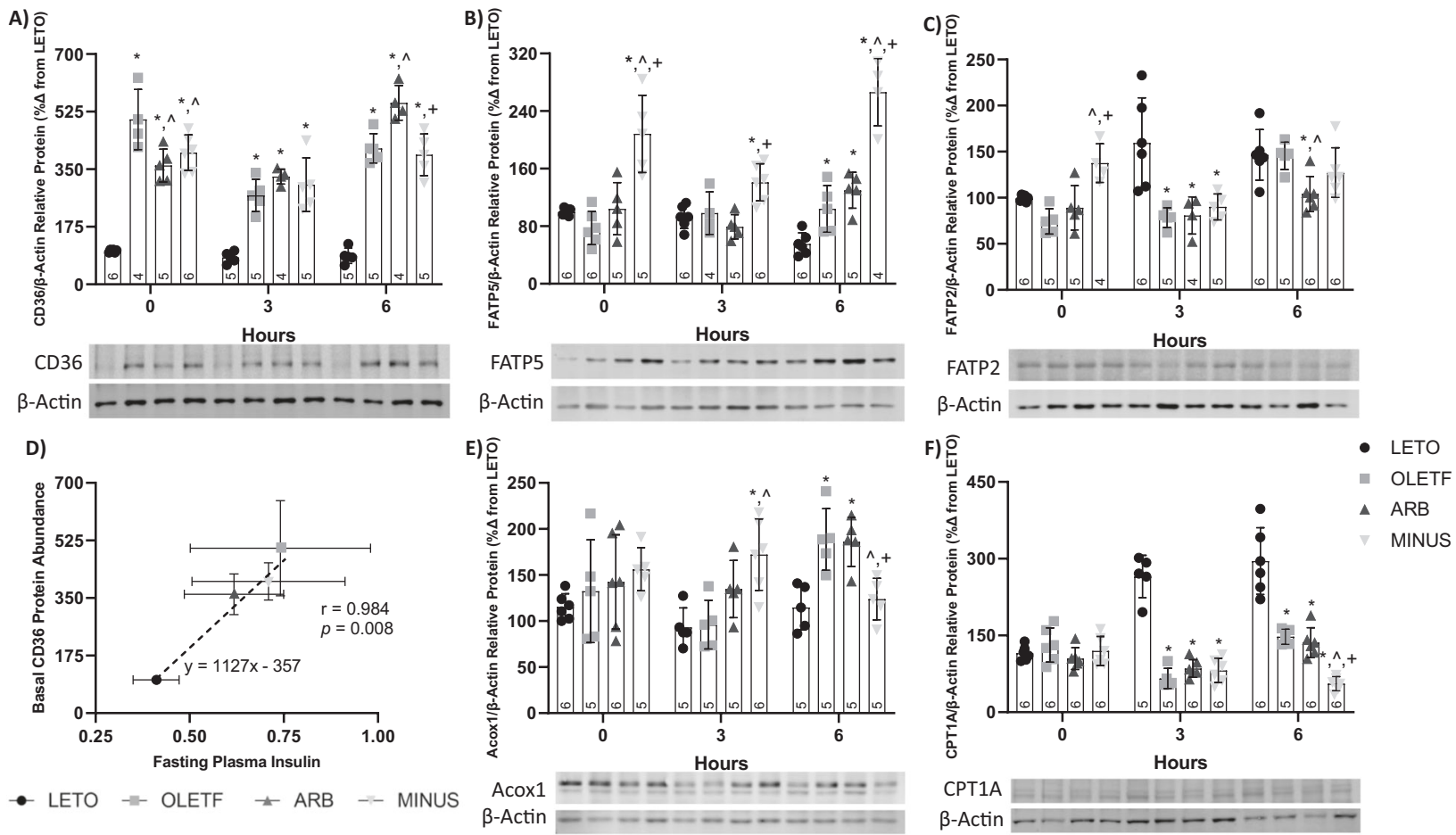

Fig. 4 ARB decreased basal membrane CD36 abundance in the liver. Western blot results, mean \pm SD values for relative liver membrane protein abundance of CD36 (A), FATP5 (B), FATP2 (C), results for Pearson's correlation test of CD36 vs Plasma Insulin (D), Acox1 (E), and CPT1A (F), during the glucose challenge in Long Evans Tokushima Otsuka (LETO), Otsuka Long Evans Tokushima Fatty (OLETF), OLETF + ARB (ARB; ARB $x 8$ weeks), and OLETF \pm ARB (MINUS, ARB x 4 weeks, then removed $x 4$ weeks) rats. Number at bottom of the bar number indicates $n$ per group. *Significant difference from LETO $(P<0.05)$. ^ Significant difference from OLETF $(P<0.05)$. ${ }^{+}$Significant difference from ARB $(P<0.05)$

(Fig. 4B). At T6, protein abundance was 47\%, 57\%, and $79 \%$ greater in OLETF, ARB and MINUS than LETO, and remained $60 \%$ and $51 \%$ greater in MINUS than OLETF and ARB, respectively (Fig. 4B).

At T3, FATP2 protein abundance was $51 \%, 50 \%$, and 43\% lesser in OLETF, ARB, and MINUS, respectively, compared to LETO (Fig. 4C). At T6, protein abundance was $29 \%$ and $28 \%$ lesser in ARB than OLETF and LETO, respectively (Fig. 4C).

Collectively, the ARB-induced decrease in basal CD36 may partially explain the amelioration in hepatic lipid accumulation [29] while the greater abundance in FATP2 and FATP5 in the MINUS group may be responsible of the increased TAG accumulation [66].

\section{Static changes}

On the other hand, Ang II signaling can increase hepatic lipids by decreasing fatty acid oxidation [5] and both Acox 1 and CPT1 are essential mediators of lipid $\beta$-oxidation $[23,67]$. We hypothesized that chronic blockade of AT1 increases $\beta$-oxidation. No basal changes in Acox 1 (Fig. 4E) or CPT1A (Fig. 4F) were detected among the groups suggesting that AT1 activation at this stage of MetS is not sufficient to profoundly impair oxidation.

\section{Dynamic changes}

During the glucose challenge, Acox1 protein abundance was $46 \%$ and $44 \%$ greater in MINUS compared to LETO and OLETF, respectively (Fig. 4D). At T6, protein abundance was 39\% and 38\% greater in OLETF and ARB than LETO, respectively, and remained $34 \%$ and $33 \%$ lesser in MINUS than OLETF and ARB, respectively (Fig. 4D).

At T3, CPT1A protein abundance was $75 \%, 68 \%$, and $81 \%$ greater in OLETF, ARB, and MINUS than LETO, respectively (Fig. 4F). At T6, protein abundance was $50 \%, 52 \%$, and $81 \%$ lesser in OLETF, ARB, and MINUS than LETO, respectively, and remained $62 \%$ and $59 \%$ lesser in MINUS than ARB and MINUS, respectively (Fig. 4F). CPT1A abundance in MINUS tended to decrease $(r=0.9944, p=0.07)$ in response to glucose over time.

The protein abundance for Acox 1 and CPT1A in MINUS remained lower than OLETF and ARB at $6 \mathrm{~h}$ following the glucose challenge suggesting that the TAG accumulation in this group was partially attributed to a reduction in lipid oxidation. This distinct behavior further demonstrates the detriment of non-compliance that is masked by analyzing static changes alone. 
Fig. 5 ARB decreased basal ApoB but not TAG synthesis protein levels in the liver.

Western blot results, mean \pm SD values for relative protein abundance of GPAM (A), DGAT1 (B), and ApoB (C), including Pearson's correlation test of ApoB vs time, during the glucose challenge in Long Evans Tokushima Otsuka (LETO), Otsuka Long Evans Tokushima Fatty (OLETF), OLETF + ARB (ARB; ARB x 8 weeks), and OLETF \pm ARB (MINUS, ARB x 4 weeks, then removed $\mathrm{x} 4$ weeks) rats.

Number at bottom of the bar number indicates $n$ per group. *Significant difference from LETO $(P<0.05))^{\wedge}$ Significant difference from OLETF $(P<$ 0.05). + Significant difference from $A R B(P<0.05)$
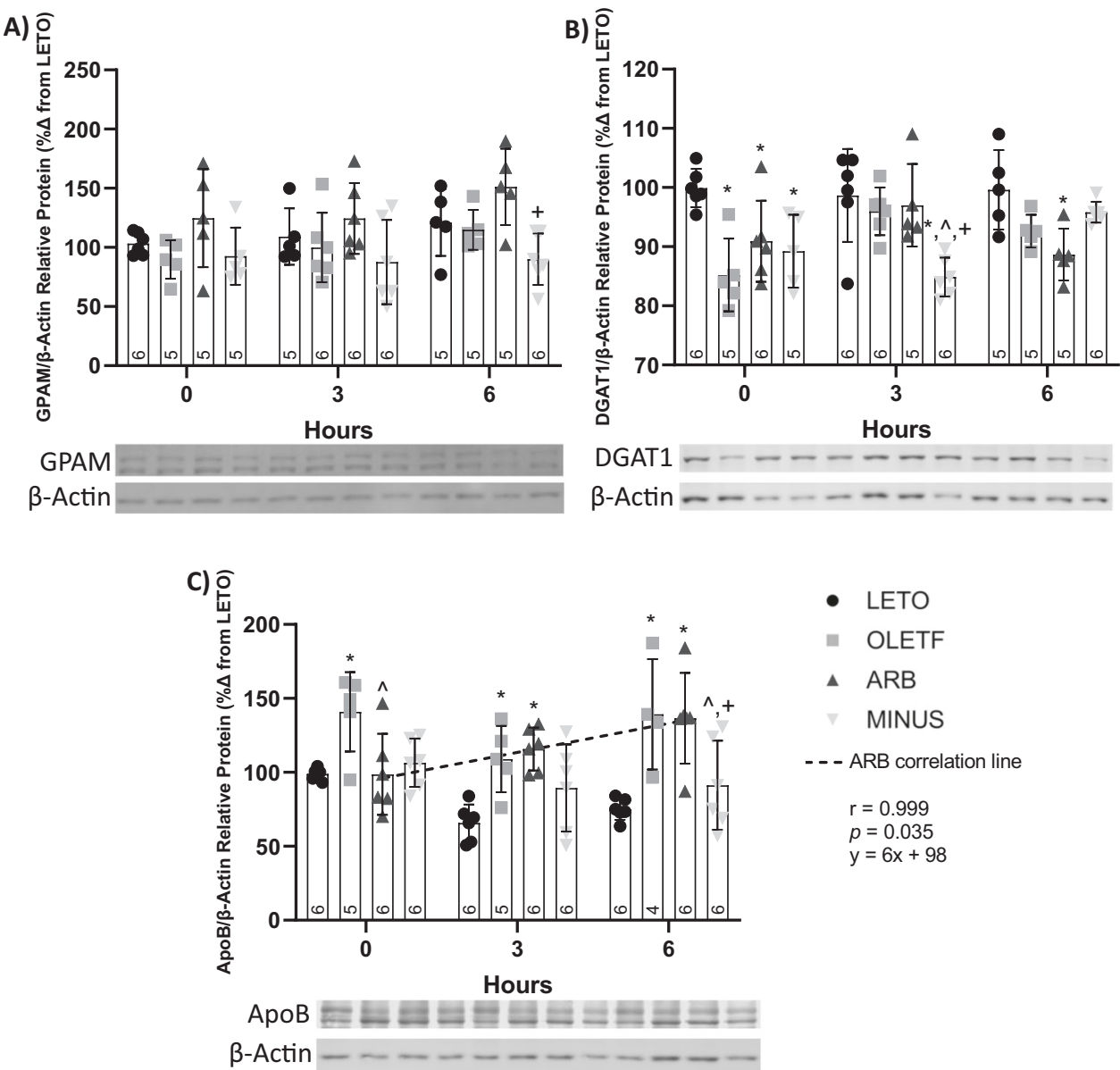

\section{ARB decreased ApoB abundance but not proteins of TAG synthesis}

Insulin can activate the liver-X-receptor, which promotes the transcription of genes of hepatic lipogenesis [68]. Previously, we demonstrated that ARB treatment decreased plasma insulin [69]. Therefore, we hypothesized that ARB treatment decreases TAG accumulation through suppression of TAG synthesis. The first committed step in TAG synthesis is catalyzed by glycerol-3-phosphate acyltransferase [70] while diglyceride acyltransferase (DGAT) mediates the last step of TAG synthesis [71].

\section{Static Changes}

In our study, basal GPAM protein abundance remained unchanged (Fig. 5A), and DGAT1 abundance was $15 \%$, $10 \%$, and $11 \%$ lesser in OLETF, ARB, and MINUS, respectively, compared to LETO (Fig. 5B).

\section{Dynamic changes}

During the glucose challenge, no profound changes in GPAM were detected among groups or over time, except for a $40 \%$ decrease in MINUS at T6 when compared to ARB (Fig. 5A). In OLETF, GPAM abundance tended $(r=0.9940, p=0.07)$ to increase in response to glucose over time. At T3, DGAT1 protein abundance was 14\%, 11\%, and $12 \%$ lesser in MINUS compared to LETO, OLETF and ARB, respectively, and at T6, was $11 \%$ lesser in ARB than LETO (Fig. 5B).

\section{Static changes}

TAGs can be used to form VLDL with ApoB, which is secreted from the liver [72] and hepatic secretion of VLDL increases with insulin resistance [73]. Therefore, we measured $A p o B$ to gain insight into hepatic VLDL formation and the effects of $\mathrm{ARB}$ on its production.

\section{Static Changes}

Basal ApoB was $29 \%$ greater in OLETF compared to LETO, and 29\% lesser in ARB than OLETF (Fig. 5C).

\section{Dynamic changes}

During the glucose challenge, ApoB protein abundance was $39 \%$ and $43 \%$ greater in OLETF and ARB, respectively, 
than LETO, at T3 (Fig. 5C). At T6, ApoB protein abundance remained $46 \%$ and $45 \%$ greater in OLETF and ARB than LETO, respectively, and was $34 \%$ and $33 \%$ lesser in MINUS compared to OLETF and ARB, respectively (Fig. 5C). ApoB levels increased linearly $(r=0.9985, p=0.04)$ over $6 \mathrm{~h}$ in ARB (Fig. 5C).

Hepatic sequestration of NEFA and chylomicron remnants is routed for oxidation or TAG esterification. TAG is also produced from excess glucose through de novo lipogenesis [5]. In our study, the abundance of TAG synthesis proteins remained unchanged with ARB treatment, while its removal caused their abundance to decrease during the glucose challenge. Elevated Ang II can alter VLDL secretion [5] and here we demonstrated that ARB treatment decreased ApoB protein abundance, a key protein for VLDL assembly [72] suggesting that less VLDL is present or being made in the liver. Furthermore, the linear increase in ApoB during the glucose challenge suggests that TAG is incorporated into VLDL to be secreted from the liver, preventing TAG accumulation.

\section{Glucose stimulates PRDX6 abundance, which was inversely correlated to hepatic TAG in ARB}

PRDX6 is a bifunctional protein with peroxidase and phospholipase A2 activity [31], which is associated with ameliorating NAFLD via its antioxidant capacity and PLA2-like activity [30, 74]. Because Ang II infusion may induce NAFLD through oxidant imbalance [6], we hypothesized that ARB treatment increases PRDX6 levels to potentially reduce oxidant imbalance.

\section{Static changes}

Basal PRDX6 protein abundance was 25\% greater in OLETF compared to LETO, and protein abundance was 45\% lesser in ARB compared to OLETF (Fig. 6A).

\section{Dynamic changes}

During the glucose challenge, PRDX6 abundance in MINUS was 30\% lesser than LETO, at T3 (Fig. 6A). At T6, protein abundance was $31 \%$ greater in OLETF compared to LETO, and $34 \%$ lesser in MINUS compared to OLETF (Fig. 6A). In ARB, PRDX6 abundance was significantly and negatively correlated with liver TAG levels $(r=$ -0.9986, $p=0.04$ ) (Fig. 6B), while PRDX6 protein abundance tended ( $r=0.9956, p=0.06)$ to positively correlate over time in response to the glucose challenge.

Notably, PRDX6 abundance was lower in the ARB group. Though the concept is new, increased PRDX6 expression protected mice from developing hepatic steatosis [30]. Accordingly, we found an inverse relationship
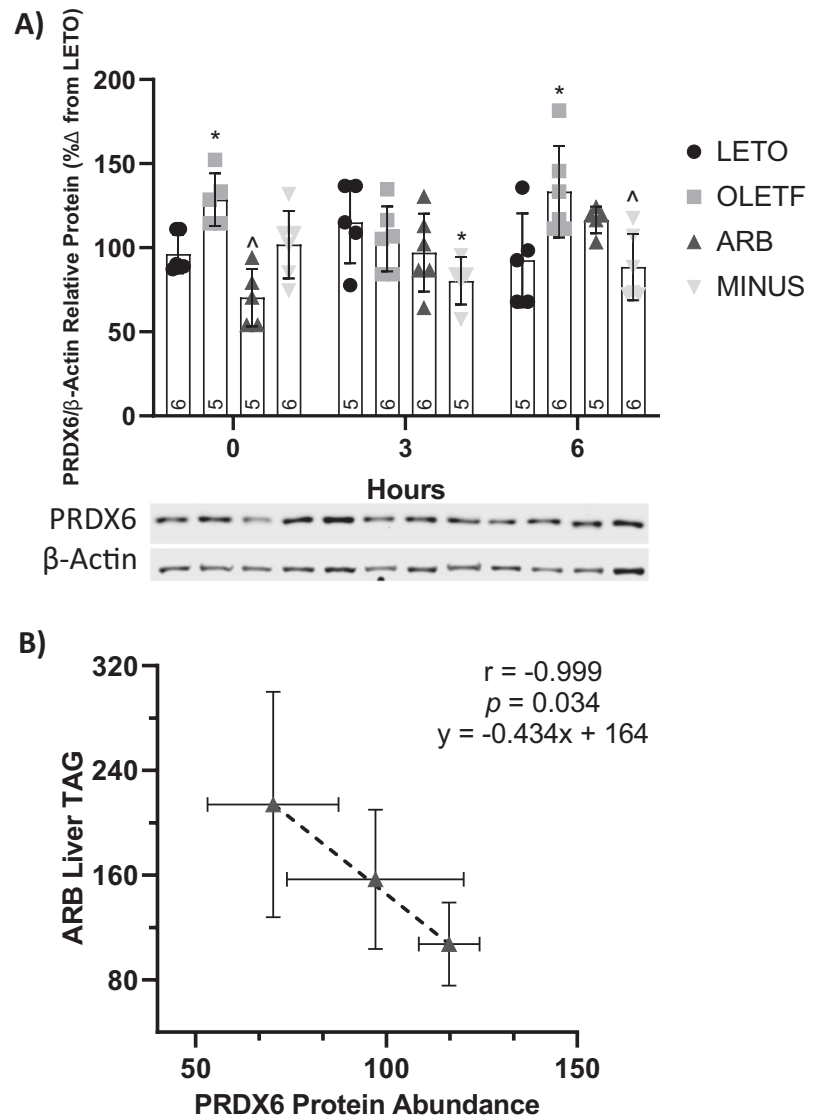

Fig. 6 Hepatic PRDX6 protein decreased with ARB, and PRDX6 abundance correlated to intrahepatic TAG. Western blot results, mean \pm SD values for relative protein abundance of PRDX6 (A) and results for Pearson's correlation test of PRDX6 vs Liver TAG (B), during the glucose challenge in Long Evans Tokushima Otsuka (LETO), Otsuka Long Evans Tokushima Fatty (OLETF), OLETF + ARB (ARB; ARB $\mathrm{x} 8$ weeks), and OLETF \pm ARB (MINUS, ARB $x 4$ weeks, then removed $x 4$ weeks) rats. Number at bottom of the bar number indicates $n$ per group. *Significant difference from LETO $(P<0.05)$. ${ }^{\wedge}$ Significant difference from OLETF $(P<0.05) .{ }^{+}$Significant difference from $A R B(P<0.05)$

between PRDX6 abundance and hepatic TAG levels, suggesting that PRDX6 abundance may present with decreased liver TAG levels and reduced hepatic steatosis. Additionally, the lower basal PRDX6 levels in ARB may reflect the lack of a need to stimulate antioxidant mechanisms, supported by improvements on redox proteins after ARB treatment $[20,36,69]$, which may be reduced as the need is ameliorated.

\section{Discussion}

NAFLD afflicts $25 \%$ of the world's population [1]. Hepatic TAG accumulation is the hallmark of NAFLD [3, 34], with lipid accumulation in the liver resulting from an imbalance between lipid sequestration and its disposal or metabolism 
[27]. Nonetheless, the mechanisms promoting steatosis in MetS [27, 35, 75] in relation to Ang II signaling [5] are not completely understood. In our study, we investigated the effects of chronic AT1 blockade statically and the dynamic responses to a glucose challenge on hepatic lipid accumulation and proteins mediating NEFA uptake, TAG and VLDL cholesterol synthesis, and fatty acid oxidation in rats with MetS. Additionally, we investigated the legacy-effect of ARB treatment following its removal. We found a marked decrease on liver TAG that may be achieved by modulating NEFA uptake, through CD36, and increased TAG export via ApoB. The inverse relationship between PRDX6 abundance and hepatic TAG and the increasing levels of PRDX6 in response to the glucose challenge when hepatic TAG levels are decreasing suggests that PRDX6 may protect the liver from steatosis derived from TAG accumulation. Furthermore, our results are unique as they highlight the detrimental effects of treatment noncompliance through many contrasts between the ARB and MINUS groups.

OLETF rats are characterized by elevated Ang II before the onset of insulin resistance [76], suggesting that elevated Ang II may contribute to the development of insulin resistance [7]. Liver-specific deletion of the Ang II receptor (AT1) reduces hepatic steatosis [63], evidencing the contribution of AT1 signaling on hepatic lipid accumulation. TAG accumulation is a hallmark of NAFLD [3] and NEFA can be a precursor for TAG formation [5], while VLDL cholesterol can be assembled into larger molecules that may not leave the hepatocyte [72]. In the liver, ARB decreased basal NEFA and TAG indicative of an improvement in liver lipid metabolism in MetS. Conversely, hepatic TAG, NEFA, and TC were increased in the MINUS group during the glucose challenge, suggesting that non-compliance is associated with hepatic lipid accumulation in response to a glucose load, consistent with a Western diet. Thus, while most of the ARB-mediated benefits remained statically, they were lost during the glucose challenge.

Hepatic expression of CD36 is typically low although it increases with fatty liver disease [27, 28]. In our study, hepatic CD36 levels in LETO were remarkably lower than all OLETF groups and remained relatively low throughout the glucose challenge. ARB treatment decreased CD36 abundance and even after its removal the levels remained lesser than OLETF. Furthermore, fasting plasma insulin and basal CD36 membrane protein abundance were strongly correlated, consistent with the data in patients with steatosis [29] suggesting that the hyperinsulinemia associated with MetS contributes to hepatic lipid accumulation and dysregulation of lipid metabolism via up-regulation of hepatic CD36.

CD36 protein abundance was increased in the myocardium of hyperglycemic mice [77] and vascular lesions of hyperglycemic patients [78], suggesting that elevated glucose may also up-regulate the abundance of CD36. In the present study, liver CD36 was greater in ARB, ending the glucose challenge. The lack of a detectable changes CD36 in response to the glucose challenge in LETO suggests that, during healthy conditions, CD36 protein levels are maintained despite the hyperglycemia. Additionally, CD36 levels decrease initially at $3 \mathrm{~h}$ post-glucose in OLETF, ARB and MINUS, increasing further only in ARB at $6 \mathrm{~h}$ postglucose, when elevated glucose levels have cleared circulation, suggesting that hepatic CD36 may not be stimulated by hyperglycemia during MetS. Thus, the changes in CD36 abundance in response to hyperglycemic conditions may be tissue-specific.

The FATP family facilitates the transport of NEFA into the cell. FATP5 and FATP2 are the most abundant in the liver [22]. Knockdown and KO studies of hepatic FATP proteins are correlated with NEFA uptake [25, 26, 79]. ARB treatment did not change the basal membrane abundance of either protein, but FATP2 abundance decreased at $6 \mathrm{~h}$ post-glucose in ARB, while CD36 was elevated in the same group, suggesting that increased CD36 may compensate for the decrease in FAT proteins in the liver. Noncompliance (MINUS) increased the FATPs expression, suggesting that the potential to sequester NEFA is increased, supported by its greater liver NEFA AUC.

Ang II-mediated signaling can increase hepatic lipidoverload by decreasing hepatic fatty acid oxidation [5]. Acox 1 is a rate-limiting enzyme in peroxisomal lipid $\beta$-oxidation [67]. Acox1-deficient rodents exhibit sudden steatosis [80]. On the other hand, CPT1A converts fatty acyl-CoA to fatty acyl-carnitine for subsequent $\beta$-oxidation [81]. Human CPT1 expression is reduced during NAFLD [82], suggesting that decreased $\beta$-oxidation may be an important factor in the development of steatosis. In our study, ARB treatment did not change Acox1 or CPT1A protein abundance; however, during the glucose challenge, Acox 1 abundance was greater in MINUS than OLETF and $\mathrm{ARB}$, while at the end of the challenge, Acox1 abundance was lesser than OLETF and ARB. These changes suggest that the removal of ARB may have changed the sensitivity of Acox 1 regulation to glucose. On the other hand, glucose induced an increase in CPT1A in the healthy LETO, which remained elevated after $6 \mathrm{~h}$, suggesting that under normal conditions a healthy liver may increase lipid oxidation to help reduce TAG and NEFA accumulation in response to a glucose load. CPT1A levels in the OLETF, ARB and MINUS groups were substantially lower than LETO, however, they increased in the OLETF and ARB groups at $6 \mathrm{~h}$ post-glucose, while MINUS levels remained suppressed. In MINUS, the decreasing trend $(p<0.07)$ in CPT1A, but not Acox1, abundance suggests that an acute glucose load may reduce the hepatic $\beta$-oxidation capabilities and that 
CPT1A abundance may be more sensitive to a glucose load, following non-compliance.

Collectively, these data suggest that: (1) under normal, healthy conditions the potential to increase $\beta$-oxidation may be primarily via CPT1-mediated mechanisms, (2) MetS may be associated with impaired CPT1A-mediated $\beta$-oxidation, which is supported by the lack of CPT1A abundance increase during the glucose challenge in the OLETF, ARB and MINUS groups, and (3) that noncompliance (MINUS) may be associated with impaired $\beta$-oxidation via CPT1A to a greater extent than untreated (OLETF) conditions.

During insulin resistance, hyperinsulinemia may increase lipogenesis and TAG accumulation [83, 84]. Insulin can increase activation of the liver-X-receptor to promote hepatic lipogenesis [68]. GPAM [70] and DGAT [71] participate in the synthesis of TAG. Hepatic GPAM expression was elevated in patients with steatosis or NASH [85]. In our study, plasma insulin AUC was lower with ARB treatment, which corresponded with lower liver TAG AUC, yet GPAM abundance remained unchanged. This may be due to GPAM activity being regulated, more so than its abundance. On the other hand, DGAT deficiency in primary hepatocytes protected against increased lipid deposition by decreasing TAG synthesis [86]. Also, inhibition of DGAT1 protected against fatty liver in mice on a high-fat diet [87]. Yet during lipodystrophy, increased de novo hepatic fatty acid synthesis caused steatosis, independent of changes in DGAT1 [88], suggesting that static changes in DGAT1 may not have a significant contribution to TAG synthesis. The increasing trend $(p<0.07)$ in GPAM abundance in response to glucose in OLETF suggests that high glucose-loads may worsen hepatic lipid accumulation by stimulating TAG synthesis. Alternatively, the measures of GPAM and DGAT protein abundance may not be enough to accurately reflect changes in the activity of these enzymes [88, 89]. The maintenance of these relatively higher levels of hepatic TAG in the OLETF and MINUS groups may be a consequence of chronically elevated TAG synthesis, which may not be stimulated by the glucose load used in this study.

Alternatively, hepatic lipids can be mobilized through the secretion of VLDLs [72], which are a primary vehicle to transport synthesized TAG to circulation for utilization in peripheral tissues. Each VLDL particle contains one molecule of ApoB [73], suggesting that ApoB abundance is a reliable surrogate measure for relative changes of VLDL in the liver. Secretion of VLDL-TAG is increased in patients with NAFLD [90]. Also, it is suggested that VLDL particles from individuals with NAFLD may be larger and contain more TAG [72, 91], preventing them from leaving the cell. Therefore, the differences in particle size cannot be excluded as a contributing factor to the observed accumulation of hepatic TAG. In our study, ApoB abundance increased in OLETF and was decreased in ARB. This suggests that the elevated levels in OLETF may be necessary to bind the greater amount of free TAG, increasing the potential for shuttling it out of the cell. Alternatively, the increased levels of ApoB in OLETF may reflect accumulation due to large particles of VLDL, which cannot be shuttled out of the liver [72].

In response to the glucose challenge, ApoB abundance increased over time in ARB. This may be due to the increased synthesis of VLDL to further decrease TAG accumulation in the liver of the ARB group. This is further supported by increased plasma TAG, which translates to elevated circulating VLDL, since VLDL accounts for $20 \%$ of the measured plasma TAG $[92,93]$. Conversely, the low abundance of ApoB in MINUS at $6 \mathrm{~h}$ post-glucose suggests that the potential to shuttle TAG out of the cell in response to glucose may be impaired, and that the increased plasma TAG may come mainly from diet and increased lipolysis, contributing to greater levels of hepatic TAG, reflecting the detrimental effect of treatment removal. Ultimately, these data provide an additional mechanism by which hepatic TAG is regulated in response to nutrient loads.

Induction of CD36 with palmitic acid induced hepatocyte activation dependent on oxidative stress pathways, while CD36 KO reduced these adverse effects [24], suggesting that changes in CD36-mediated NEFA transport are associated with changes in redox balance in the liver. However, these relationships are not well-defined during MetS. PRDX6 is member of the PRDX family of proteins, which may protect against obesity-related pathologies, mainly through elimination of oxidants [94-96]. The C57BL/6J-Tg transgenic mice with increased PRDX6 expression prevented the liver from developing steatosis [30], suggesting that PRDX6 may contribute directly to the regulation of hepatic lipid accumulation. PRDX6 KO mice on a high-fat diet increased levels of circulating alanine aminotransferase (ALT), a marker of hepatic injury that is associated with development of NASH [74]. In our study, basal PRDX6 abundance was greater in OLETF, while ARB treatment reduced this increase, suggesting that $A R B$ treatment improved the redox status in the liver, and that increased levels of hepatic PRDX6 may reflect the need for improved redox balance in the liver. Glucose stimulated a trend $(p<$ 0.06) for linear increase in the ARB group, suggesting that a glucose load may stimulate redox gene transcription factors to initiate antioxidant mechanisms in response to increased glucose levels. PRDX6 and liver TAG levels were negatively correlated in the ARB group, suggesting that either: (a) increasing hepatic TAG accumulation may decrease PRDX6 levels or (b) increasing PRDX6 reduces hepatic TAG accumulation. Yet, very limited information is available regarding the relationship between PRDX6 and liver 
TAG levels. Therefore, in the current study, deciphering which is the independent and dependent variable is not possible, though the novel finding is that there is a negative relationship between these two variables that has not been previously reported in a model of MetS. Although the negative correlation between PRDX6 and liver TAG can be thought as isolated, it may be meaningful for future research linking PRDX6 and NAFLD, where literature on this topic is scarce. Although the link between NAFLD and PRDX6 remains unclear [30,74], our data supports the idea that PRDX6 may participate in protecting the liver from TAG accumulation.

In summary, these results demonstrate that chronic blockade of AT1, through ARB treatment, protects the liver from TAG accumulation, especially during a glucose load. This may be achieved by decreasing NEFA uptake and increasing TAG export via CD36 and FATP2, and ApoB, respectively. Additionally, treatment non-compliance reverted many of the potential benefits observed in ARB, which may leave the liver more susceptible to further lipid accumulation and injury over time. Finally, frequent acute glucose loads may contribute to increased hepatic lipid accumulation through the maintenance of TAG synthesis and impaired $\beta$-oxidation and cellular lipid export, to ultimately develop NAFLD in MetS.

\section{Data availability}

Associated data are available upon reasonable request.

Author contributions Conceptualization, project administration, and supervision: M.A.T., J.A.G.L., A.N., D.N., J.G.S.O., R.M.O. Investigation: J.A.G.L., D.Y.H. Data curation: J.A.G.L., R.M.O. Writing, original draft preparation: J.A.G.L. Writing, reviewing, and editing: J.A.G.L., M.A.T., D.Y.H., A.N., D.N., J.G.S.O., R.M.O.

Funding Research was partially funded by University of California Mexico-United States (UC MEXUS) grant 19-194. J.A.G.L. was supported by the UC-MEXUS Consejo Nacional de Ciencia y Tecnologia (CONACYT) fellowship. J.A.G.L. and M.A.T. were supported by internal funding from University of California, Merced and by NIH NCMHD9T37MD001480.

\section{Compliance with ethical standards}

Conflict of interest The authors declare no competing interests.

Ethical approval All experimental procedures were reviewed and approved by the institutional animal care and use committees of the Kagawa Medical University (Japan) and the University of California, Merced (USA).

Publisher's note Springer Nature remains neutral with regard to jurisdictional claims in published maps and institutional affiliations.

Open Access This article is licensed under a Creative Commons Attribution 4.0 International License, which permits use, sharing, adaptation, distribution and reproduction in any medium or format, as long as you give appropriate credit to the original author(s) and the source, provide a link to the Creative Commons license, and indicate if changes were made. The images or other third party material in this article are included in the article's Creative Commons license, unless indicated otherwise in a credit line to the material. If material is not included in the article's Creative Commons license and your intended use is not permitted by statutory regulation or exceeds the permitted use, you will need to obtain permission directly from the copyright holder. To view a copy of this license, visit http://creativecommons. org/licenses/by/4.0/.

\section{References}

1. T.G. Cotter, M. Rinella, Nonalcoholic fatty liver disease 2020: the state of the disease. Gastroenterology 158, 1851-1864 (2020)

2. D.J. Chiang, M.T. Pritchard, L.E. Nagy, Obesity, diabetes mellitus, and liver fibrosis. Am J Physiol Gastrointest Liver Physiol 300, G697-G702 (2011)

3. P. Paschos, K. Paletas, Non alcoholic fatty liver disease and metabolic syndrome. Hippokratia 13, 9-19 (2009)

4. S.M. Grundy, J.I. Cleeman, S.R. Daniels, K.A. Donato, R.H. Eckel et al. Diagnosis and management of the metabolic syndrome: an American Heart Association/National Heart, Lung, and Blood Institute Scientific Statement. Circulation 112, 2735-2752 (2005)

5. E. Matthew Morris, J.A. Fletcher, J.P. Thyfault, R.S. Rector, The role of angiotensin II in nonalcoholic steatohepatitis. Mol Cell Endocrinol 378, 29-40 (2013)

6. Y. Wei, S.E. Clark, E.M. Morris, J.P. Thyfault, G.M. Uptergrove et al. Angiotensin II-induced non-alcoholic fatty liver disease is mediated by oxidative stress in transgenic TG(mRen2)27(Ren2) rats. J Hepatol 49, 417-428 (2008)

7. J.A. Olivares-Reyes, A. Arellano-Plancarte, J.R. Castillo-Hernandez, Angiotensin II and the development of insulin resistance: implications for diabetes. Mol Cell Endocrinol 302, 128-139 (2009)

8. E.J. Henriksen, S. Jacob, T.R. Kinnick, M.K. Teachey, M. Krekler, Selective angiotensin II receptor antagonism reduces insulin resistance in obese Zucker rats. Hypertension 38, 884-890 (2001)

9. J.A. Sloniger, V. Saengsirisuwan, C.J. Diehl, J.S. Kim, E.J. Henriksen, Selective angiotensin II receptor antagonism enhances wholebody insulin sensitivity and muscle glucose transport in hypertensive TG(mREN2)27 rats. Metabolism 54, 1659-1668 (2005)

10. Y. Li, F. Xiong, W. Xu, S. Liu, Increased serum angiotensin II is a risk factor of nonalcoholic fatty liver disease: a prospective pilot study. Gastroenterol Res Pract 2019, 5647161 (2019)

11. J. Ran, T. Hirano, M. Adachi, Chronic ANG II infusion increases plasma triglyceride level by stimulating hepatic triglyceride production in rats. Am J Physiol Endocrinol Metab 287, E955-E961 (2004)

12. J. Ran, T. Hirano, M. Adachi, Angiotensin II type 1 receptor blocker ameliorates overproduction and accumulation of triglyceride in the liver of Zucker fatty rats. Am J Physiol Endocrinol Metab 287, E227-E232 (2004)

13. A. Barreras, C. Gurk-Turner, Angiotensin II receptor blockers. Proc Bayl Univ Med Cent 16, 123-126 (2003)

14. S. Yamada, Pleiotropic effects of ARB in metabolic syndrome. Curr Vasc Pharmacol 9, 158-161 (2011)

15. Y. Li, H. Xu, W. Wu, J. Ye, D. Fang et al. Clinical application of angiotensin receptor blockers in patients with non-alcoholic fatty liver disease: a systematic review and meta-analysis. Oncotarget $\mathbf{9}$, 24155-24167 (2018) 
16. L.M.A. Borem, J.F.R. Neto, I.V. Brandi, D.F. Lelis, S.H.S. Santos, The role of the angiotensin II type I receptor blocker telmisartan in the treatment of non-alcoholic fatty liver disease: a brief review. Hypertens Res 41, 394-405 (2018)

17. S.M. Kyvelou, G.P. Vyssoulis, E.A. Karpanou, D.N. Adamopoulos, A.I. Zervoudaki et al. Effects of antihypertensive treatment with angiotensin II receptor blockers on lipid profile: an open multi-drug comparison trial. Hellenic J Cardiol 47, 21-28 (2006)

18. M. Hanefeld, C. Abletshauser, Effect of the angiotensin II receptor antagonist valsartan on lipid profile and glucose metabolism in patients with hypertension. J Int Med Res 29, 270-279 (2001)

19. I. Taguchi, S. Toyoda, K. Takano, T. Arikawa, M. Kikuchi et al. Irbesartan, an angiotensin receptor blocker, exhibits metabolic, anti-inflammatory and antioxidative effects in patients with highrisk hypertension. Hypertens Res 36, 608-613 (2013)

20. P. Montez, J.P. Vazquez-Medina, R. Rodriguez, M.A. Thorwald, J.A. Viscarra et al. Angiotensin receptor blockade recovers hepatic UCP2 expression and aconitase and SDH activities and ameliorates hepatic oxidative damage in insulin resistant rats. Endocrinology 153, 5746-5759 (2012)

21. K. Kaji, H. Yoshiji, M. Kitade, Y. Ikenaka, R. Noguchi et al. Combination treatment of angiotensin II type I receptor blocker and new oral iron chelator attenuates progression of nonalcoholic steatohepatitis in rats. Am J Physiol Gastrointest Liver Physiol 300, G1094-G1104 (2011)

22. D. Hirsch, A. Stahl, H.F. Lodish, A family of fatty acid transporters conserved from mycobacterium to man. Proc Natl Acad Sci USA 95, 8625-8629 (1998)

23. S.H. Koo, Nonalcoholic fatty liver disease: molecular mechanisms for the hepatic steatosis. Clin Mol Hepatol 19, 210-215 (2013)

24. J. Liu, P. Yang, G. Zuo, S. He, W. Tan et al. Long-chain fatty acid activates hepatocytes through CD36 mediated oxidative stress. Lipids Health Dis 17, 153 (2018)

25. A. Falcon, H. Doege, A. Fluitt, B. Tsang, N. Watson et al. FATP2 is a hepatic fatty acid transporter and peroxisomal very long-chain acyl-CoA synthetase. Am J Physiol Endocrinol Metab 299, E384-E393 (2010)

26. H. Doege, R.A. Baillie, A.M. Ortegon, B. Tsang, Q. Wu et al. Targeted deletion of FATP5 reveals multiple functions in liver metabolism: alterations in hepatic lipid homeostasis. Gastroenterology 130, 1245-1258 (2006)

27. D.H. Ipsen, J. Lykkesfeldt, P. Tveden-Nyborg, Molecular mechanisms of hepatic lipid accumulation in non-alcoholic fatty liver disease. Cell Mol Life Sci 75, 3313-3327 (2018)

28. M. Inoue, T. Ohtake, W. Motomura, N. Takahashi, Y. Hosoki et al. Increased expression of PPARgamma in high fat dietinduced liver steatosis in mice. Biochem Biophys Res Commun 336, 215-222 (2005)

29. M.E. Miquilena-Colina, E. Lima-Cabello, S. Sanchez-Campos, M. V. Garcia-Mediavilla, M. Fernandez-Bermejo et al. Hepatic fatty acid translocase CD36 upregulation is associated with insulin resistance, hyperinsulinaemia and increased steatosis in nonalcoholic steatohepatitis and chronic hepatitis C. Gut 60, 1394-1402 (2011)

30. D.H. Lee, Y.Y. Jung, M.H. Park, M.R. Jo, S.B. Han et al. Peroxiredoxin 6 confers protection against nonalcoholic fatty liver disease through maintaining mitochondrial function. Antioxid Redox Signal 31, 387-402 (2019)

31. A.B. Fisher, Peroxiredoxin 6: a bifunctional enzyme with glutathione peroxidase and phospholipase $\mathrm{A}(2)$ activities. Antioxid Redox Signal 15, 831-844 (2011)

32. T. Eismann, N. Huber, T. Shin, S. Kuboki, E. Galloway et al. Peroxiredoxin-6 protects against mitochondrial dysfunction and liver injury during ischemia-reperfusion in mice. Am J Physiol Gastrointest Liver Physiol 296, G266-G274 (2009)
33. I.C.M. Simoes, A. Fontes, P. Pinton, H. Zischka, M.R. Wieckowski, Mitochondria in non-alcoholic fatty liver disease. Int $\mathrm{J}$ Biochem Cell Biol 95, 93-99 (2018)

34. C.J. Green, C. Pramfalk, K.J. Morten, L. Hodson, From whole body to cellular models of hepatic triglyceride metabolism: man has got to know his limitations. Am J Physiol Endocrinol Metab 308, E1-E20 (2015)

35. N. Alkhouri, L.J. Dixon, A.E. Feldstein, Lipotoxicity in nonalcoholic fatty liver disease: not all lipids are created equal. Expert Rev Gastroenterol Hepatol 3, 445-451 (2009)

36. M.A. Thorwald, J.A. Godoy-Lugo, G.J. Rodriguez, M.A. Rodriguez, M. Jamal et al. Nrf2-related gene expression is impaired during a glucose challenge in type II diabetic rat hearts. Free Radic Biol Med 130, 306-317 (2019)

37. W. Kopp. How Western diet and lifestyle drive the pandemic of obesity and civilization diseases. Diabetes Metab Syndr Obes 12, 2221-36 (2019).

38. S. Moreno-Fernandez, M. Garces-Rimon, G. Vera, J. Astier, J.F. Landrier et al. High fat/high glucose diet induces metabolic syndrome in an experimental rat model. Nutrients 10, 1-15 (2018)

39. H. Sasamura, H. Itoh, 'Memory' and 'legacy' in hypertension and lifestyle-related diseases. Hypertens Res 35, 272-273 (2012)

40. N. Lopes, A.C. Zanini, A. Casella-Filho, A.C. Chagas, Metabolic syndrome patient compliance with drug treatment. Clinics 63, 573-580 (2008)

41. E.A. Alefishat, R.K. Abu Farha, M.M. Al-Debei, Self-reported adherence among individuals at high risk of metabolic syndrome: effect of knowledge and attitude. Med Princ Pract 26, 157-163 (2017)

42. N. Togashi, T. Maeda, H. Yoshida, M. Koyama, M. Tanaka et al. Angiotensin II receptor activation in youth triggers persistent insulin resistance and hypertension-a legacy effect? Hypertens Res 35, 334-340 (2012)

43. M. Volpe, F. Cosentino, G. Tocci, F. Palano, F. Paneni, Antihypertensive therapy in diabetes: the legacy effect and RAAS blockade. Curr Hypertens Rep 13, 318-324 (2011)

44. K. Kawano, T. Hirashima, S. Mori, T. Natori, OLETF (Otsuka Long-Evans Tokushima Fatty) rat: a new NIDDM rat strain. Diabetes Res Clin Pract 24, S317-S320 (1994)

45. K. Kawano, T. Hirashima, S. Mori, Y. Saitoh, M. Kurosumi et al. Spontaneous long-term hyperglycemic rat with diabetic complications. Otsuka Long-Evans Tokushima Fatty (OLETF) strain. Diabetes 41, 1422-1428 (1992)

46. H.R. Brunner, Olmesartan medoxomil: current status of its use in monotherapy. Vasc Health Risk Manag 2, 327-340 (2006)

47. A. Ghasemi, S. Zahediasl, Normality tests for statistical analysis: a guide for non-statisticians. Int J Endocrinol Metab 10, 486-489 (2012)

48. M.M. Mukaka, Statistics corner: a guide to appropriate use of correlation coefficient in medical research. Malawi Med J 24, 69-71 (2012)

49. P. Zabielski, A. Blachnio-Zabielska, I.R. Lanza, S. Gopala, S. Manjunatha et al. Impact of insulin deprivation and treatment on sphingolipid distribution in different muscle subcellular compartments of streptozotocin-diabetic C57B1/6 mice. Am J Physiol Endocrinol Metab 306, E529-E542 (2014)

50. T. Jaki, M.J. Wolfsegger, A theoretical framework for estimation of AUCs in complete and incomplete sampling designs. Statistics in Biopharmaceutical Research 1, 176-184 (2009)

51. H. Heikela, S.T. Ruohonen, M. Adam, R. Viitanen, H. Liljenback et al. Hydroxysteroid (17beta) dehydrogenase 12 is essential for metabolic homeostasis in adult mice. Am J Physiol Endocrinol Metab 319, E494-E508 (2020)

52. S.K. Kwak, J.H. Kim, Statistical data preparation: management of missing values and outliers. Korean J Anesthesiol 70, 407-411 (2017) 
53. H. Muller-Fielitz, N. Hubel, M. Mildner, F.M. Vogt, J. Barkhausen et al. Chronic blockade of angiotensin AT(1) receptors improves cardinal symptoms of metabolic syndrome in dietinduced obesity in rats. Br J Pharmacol 171, 746-760 (2014)

54. N. Kitamura, Y. Takahashi, S. Yamadate, S. Asai, Angiotensin II receptor blockers decreased blood glucose levels: a longitudinal survey using data from electronic medical records. Cardiovasc Diabetol 6, 26 (2007)

55. J. Yao, X. Gong, X. Shi, S. Fan, J. Chen et al. The efficacy of angiotensin converting enzyme inhibitors versus angiotensin II receptor blockers on insulin resistance in hypertensive patients: a protocol for a systematic review and meta-analysis. Medicine 99, e20674 (2020)

56. T. Sasaki, Y. Noda, Y. Yasuoka, H. Irino, H. Abe et al. Comparison of the effects of telmisartan and olmesartan on home blood pressure, glucose, and lipid profiles in patients with hypertension, chronic heart failure, and metabolic syndrome. Hypertens Res 31, 921-929 (2008)

57. L. Ciccarelli, Angiotensin II receptor blockers and insulin resistance. Hypertens Res 33, 779 (2010)

58. J.T. Stefano, L.V. Guedes, A.A.A. de Souza, D.S. Vanni, V.A.F. Alves et al. Usefulness of collagen type IV in the detection of significant liver fibrosis in nonalcoholic fatty liver disease. Ann Hepatol 20, 100253 (2021)

59. M. Mizuno, T. Shima, H. Oya, Y. Mitsumoto, C. Mizuno et al. Classification of patients with non-alcoholic fatty liver disease using rapid immunoassay of serum type IV collagen compared with liver histology and other fibrosis markers. Hepatol Res 47, 216-225 (2017)

60. H. Takagi, T. Umemoto, Telmisartan reduces triglyceride levels over other angiotensin II receptor blockers: a meta-analysis of randomized head-to-head trials. Int J Cardiol 157, 403-407 (2012)

61. Y.S. Song, C.H. Fang, B.I. So, J.Y. Park, Y. Lee et al. Time course of the development of nonalcoholic Fatty liver disease in the Otsuka long-evans Tokushima Fatty rat. Gastroenterol Res Pract 2013, 342648 (2013)

62. M.Z. Teixeira, Rebound effect of modern drugs: serious adverse event unknown by health professionals. Rev Assoc Med Bras 59, 629-638 (2013)

63. Y. Nabeshima, S. Tazuma, K. Kanno, H. Hyogo, K. Chayama, Deletion of angiotensin II type I receptor reduces hepatic steatosis. J Hepatol 50, 1226-1235 (2009)

64. M.Y. Pepino, O. Kuda, D. Samovski, N.A. Abumrad, Structurefunction of CD36 and importance of fatty acid signal transduction in fat metabolism. Annu Rev Nutr 34, 281-303 (2014)

65. C.M. Anderson, A. Stahl, SLC27 fatty acid transport proteins. Mol Aspects Med 34, 516-528 (2013)

66. K. Enooku, T. Tsutsumi, M. Kondo, N. Fujiwara, T. Sasako et al. Hepatic FATP5 expression is associated with histological progression and loss of hepatic fat in NAFLD patients. J Gastroenterol 55, 227-243 (2020)

67. M.E. Moreno-Fernandez, D.A. Giles, T.E. Stankiewicz, R. Sheridan, R. Karns et al. Peroxisomal beta-oxidation regulates whole body metabolism, inflammatory vigor, and pathogenesis of nonalcoholic fatty liver disease. JCI Insight 3, 1-19 (2018)

68. G. Chen, G. Liang, J. Ou, J.L. Goldstein, M.S. Brown, Central role for liver $\mathrm{X}$ receptor in insulin-mediated activation of Srebp-1c transcription and stimulation of fatty acid synthesis in liver. Proc Natl Acad Sci USA 101, 11245-11250 (2004)

69. M. Thorwald, R. Rodriguez, A. Lee, B. Martinez, J. Peti-Peterdi et al. Angiotensin receptor blockade improves cardiac mitochondrial activity in response to an acute glucose load in obese insulin resistant rats. Redox Biol 14, 371-378 (2018)

70. J. Yu, K. Loh, Z.Y. Song, H.Q. Yang, Y. Zhang et al. Update on glycerol-3-phosphate acyltransferases: the roles in the development of insulin resistance. Nutr Diabetes 8, 34 (2018)
71. C.L. Yen, S.J. Stone, S. Koliwad, C. Harris, R.V. Farese Jr, Thematic review series: glycerolipids DGAT enzymes and triacylglycerol biosynthesis. J Lipid Res 49, 2283-2301 (2008)

72. F.M. Perla, M. Prelati, M. Lavorato, D. Visicchio, C. Anania, The role of lipid and lipoprotein metabolism in non-alcoholic fatty liver disease. Children 4, 1-14 (2017)

73. S.H. Choi, H.N. Ginsberg, Increased very low density lipoprotein (VLDL) secretion, hepatic steatosis, and insulin resistance. Trends Endocrinol Metab 22, 353-363 (2011)

74. R. Arriga, F. Pacifici, B. Capuani, A. Coppola, A. Orlandi et al. Peroxiredoxin 6 is a key antioxidant enzyme in modulating the link between glycemic and lipogenic metabolism. Oxid Med Cell Longev 2019, 9685607 (2019)

75. A. McCullough, S.F. Previs, J. Dasarathy, K. Lee, A. Osme et al. HDL flux is higher in patients with nonalcoholic fatty liver disease. Am J Physiol Endocrinol Metab 317, E852-E862 (2019)

76. Y. Nagai, L. Yao, H. Kobori, K. Miyata, Y. Ozawa et al. Temporary angiotensin II blockade at the prediabetic stage attenuates the development of renal injury in type 2 diabetic rats. J Am Soc Nephrol 16, 703-711 (2005)

77. D.E. Greenwalt, S.H. Scheck, T. Rhinehart-Jones, Heart CD36 expression is increased in murine models of diabetes and in mice fed a high fat diet. J Clin Invest 96, 1382-1388 (1995)

78. E. Griffin, A. Re, N. Hamel, C. Fu, H. Bush et al. A link between diabetes and atherosclerosis: glucose regulates expression of CD36 at the level of translation. Nat Med 7, 840-846 (2001)

79. H. Doege, D. Grimm, A. Falcon, B. Tsang, T.A. Storm et al. Silencing of hepatic fatty acid transporter protein 5 in vivo reverses diet-induced non-alcoholic fatty liver disease and improves hyperglycemia. J Biol Chem 283, 22186-22192 (2008)

80. K. Meyer, Y. Jia, W.Q. Cao, P. Kashireddy, M.S. Rao, Expression of peroxisome proliferator-activated receptor alpha, and PPARalpha regulated genes in spontaneously developed hepatocellular carcinomas in fatty acyl-CoA oxidase null mice. Int $\mathbf{J}$ Oncol 21, 1175-1180 (2002)

81. S. Eaton, K. Bartlett, M. Pourfarzam, Mammalian mitochondrial beta-oxidation. Biochem J 320, 345-357 (1996)

82. M. Kohjima, M. Enjoji, N. Higuchi, M. Kato, K. Kotoh et al. Reevaluation of fatty acid metabolism-related gene expression in nonalcoholic fatty liver disease. Int $\mathbf{J}$ Mol Med 20, 351-358 (2007)

83. R.A. Coleman, D.P. Lee, Enzymes of triacylglycerol synthesis and their regulation. Prog Lipid Res 43, 134-176 (2004)

84. C.A. Nagle, E.L. Klett, R.A. Coleman, Hepatic triacylglycerol accumulation and insulin resistance. J Lipid Res 50, S74-S79 (2009)

85. B.M. Arendt, E.M. Comelli, D.W. Ma, W. Lou, A. Teterina et al. Altered hepatic gene expression in nonalcoholic fatty liver disease is associated with lower hepatic n-3 and n-6 polyunsaturated fatty acids. Hepatology 61, 1565-1578 (2015)

86. C.J. Villanueva, M. Monetti, M. Shih, P. Zhou, S.M. Watkins et al. Specific role for acyl CoA:Diacylglycerol acyltransferase 1 (Dgat1) in hepatic steatosis due to exogenous fatty acids. Hepatology 50, 434-442 (2009)

87. S.J. Smith, S. Cases, D.R. Jensen, H.C. Chen, E. Sande et al. Obesity resistance and multiple mechanisms of triglyceride synthesis in mice lacking Dgat. Nat Genet 25, 87-90 (2000)

88. I. Shimomura, R.E. Hammer, J.A. Richardson, S. Ikemoto, Y. Bashmakov et al. Insulin resistance and diabetes mellitus in transgenic mice expressing nuclear SREBP-1c in adipose tissue: model for congenital generalized lipodystrophy. Genes Dev 12, 3182-3194 (1998)

89. T. Kodama, J. Yi, J.Y. Newberg, J.C. Tien, H. Wu et al. Molecular profiling of nonalcoholic fatty liver disease-associated hepatocellular carcinoma using SB transposon mutagenesis. Proc Natl Acad Sci USA 115, E10417-E10426 (2018) 
90. E. Fabbrini, B.S. Mohammed, F. Magkos, K.M. Korenblat, B.W. Patterson et al. Alterations in adipose tissue and hepatic lipid kinetics in obese men and women with nonalcoholic fatty liver disease. Gastroenterology 134, 424-431 (2008)

91. J.D. Horton, H. Shimano, R.L. Hamilton, M.S. Brown, J.L. Goldstein, Disruption of LDL receptor gene in transgenic SREBP1a mice unmasks hyperlipidemia resulting from production of lipid-rich VLDL. J Clin Invest 103, 1067-1076 (1999)

92. R.A. Cox, M.R. Garcia-Palmieri. Cholesterol, triglycerides, and associated lipoproteins. In: H.K. Walker, W.D. Hall, J.W. Hurst (eds) Clinical methods: the history, physical, and laboratory examinations (Butterworth Publishers, Boston, 1990)

93. K.R. Feingold. Introduction to Lipids and Lipoproteins. In: K.R. Feingold, B. Anawalt, A. Boyce, G. Chrousos, W.W. de Herder, et al. (eds) Endotext. (MDText.com, Inc., South Dartmouth, MA, 2000)

94. A. Nabeshima, S. Yamada, X. Guo, A. Tanimoto, K.Y. Wang et al. Peroxiredoxin 4 protects against nonalcoholic steatohepatitis and type 2 diabetes in a nongenetic mouse model. Antioxid Redox Signal 19, 1983-1998 (2013)

95. G.M. Schmid, V. Converset, N. Walter, M.V. Sennitt, K.Y. Leung et al. Effect of high-fat diet on the expression of proteins in muscle, adipose tissues, and liver of C57BL/6 mice. Proteomics 4, 2270-2282 (2004)

96. J.Y. Huh, Y. Kim, J. Jeong, J. Park, I. Kim et al. Peroxiredoxin 3 is a key molecule regulating adipocyte oxidative stress, mitochondrial biogenesis, and adipokine expression. Antioxid Redox Signal 16, 229-243 (2012) 